\title{
Enhanced Disturbance-Observer-Based Control for a Class of Time-Delay System with Uncertain Sinusoidal Disturbances
}

\author{
Xinyu Wen \\ School of Electronic Information Engineering, Taiyuan University of Science and Technology, Taiyuan 030024, China \\ Correspondence should be addressed to Xinyu Wen; wxysunrain@163.com
}

Received 6 February 2013; Accepted 1 April 2013

Academic Editor: Yang Yi

Copyright (C) 2013 Xinyu Wen. This is an open access article distributed under the Creative Commons Attribution License, which permits unrestricted use, distribution, and reproduction in any medium, provided the original work is properly cited.

\begin{abstract}
This paper is concerned with disturbance-observer-based control (DOBC) for a class of time-delay systems with uncertain sinusoidal disturbances. The disturbances are decomposed as precise and uncertain parts using nonlinear disturbance observer (DO) after appropriate coordinate transformation. And then the two parts can be compensated by corresponding controller, respectively, such that the classic DOBC method is extended to uncertain disturbance rejection. One novel feature of the proposed method is that even if the precise disturbance parameters are inaccessible, the merits of DOBC can be inherited. By integrating the disturbance observers with feedback control laws with time delay, the disturbances can be rejected and the desired dynamic performances can be guaranteed. Finally, simulations for a flight control system are given to demonstrate the effectiveness of the results.
\end{abstract}

\section{Introduction}

Dynamical systems with time delays [1-3] widely exist in many systems, such as hydraulic processes, chemical systems, and temperature processes. In addition, the presence of exogenous disturbances is inevitable in engineering control systems; a complex system may suffer various of disturbances due to inherent physical property including sensor measurement noise, control error, and structural vibrations. As the phenomenons mentioned above are often primary sources of instability and performance degradation, it is an impendence thing to design control strategy for time-delay systems characteristic with antidisturbance performance. Some researchers [4-6] have contributed on this subject recently, in $[4,5]$ the $H_{\infty}$ control is adopted to attenuate the influences from disturbances to a desired level, for systems with the bounded disturbances. In [6] a reduced-order observer is structured for the estimation of the modeled disturbance; simultaneously, $H_{\infty}$ scheme can attenuate norm bounded signals. Due to the increasing complexity of the controlled plants and environment, it makes the higher demand for system accuracy, reliability, and real-time performance.

Disturbance-observer-based control (DOBC) is a prevalent anti-disturbance control strategy, which has a simple structure and is easily implemental in engineering (see surveys [7] and references therein). If the priori characteristics of disturbance to be estimated can be obtained, DOBC can be implemented where the disturbance compensation dynamic property within a composite system can be analyzed [811]. Originating from [9], a hierarchical control strategy is established in $[6,10,11]$ aiming at multiple disturbances in multiinput multioutput (MIMO) nonlinear system; the outcome shows that the strategy has high precision together with strong robustness. The literature mentioned above shows that the DOBC is feasible for more complex structure and can avoid heavy computation, such as resolve of partial differential equations (PDEs) compared with output regulation theory. However, the main limitation of the classical DOBC is that the precise characteristic parameters of disturbance must be available. Moreover, failure in modeling for disturbance accurately may lead to severe deterioration of closed loop system performance, even to instability. It has not been reported that DOBC is presented for time-delay systems subject to uncertain disturbances.

In DOBC [9-11, 13], the disturbance is seen as extended state, correspondingly an extended state observer; that is, disturbance observer (DO) can be constructed to estimate the disturbance. Once we have no access of the precise 
disturbance model, no effective observer can be constructed directly to estimate the disturbance as the matching condition [12] is not satisfied. It still remains challenging work to extend the DOBC to the general case, in which the disturbance dynamic model has parametric uncertainty The aim of this paper is to provide a novel approach to estimate and reject the uncertain disturbances, such that the merits of DOBC can be inherited. We first construct an auxiliary observer and then decompose the disturbances into a known precise function, an uncertain nonlinear function, and a decaying vector defined by the auxiliary observer. Corresponding disturbance rejection strategy can be implemented to deal with the uncertain disturbance after the sophisticated design with lower conservativeness compared with the literature mentioned above.

The organization of the problem is given below. Section 2 gives the problem formulation. In Section 3, the formulation for the uncertain disturbance estimation with time delay is introduced. In Section 4, by using the auxiliary vector, DOBC combined with adaptive controller is designed to reject the disturbance and globally stabilize the closed-loop systems. In Section 5, the proposed method is applied to an A4D aircraft model; simulations show the effectiveness of the proposed approaches. Section 6 provides conclusions.

\section{Formulation of the Problem}

The following continuous time-delay system with uncertain perturbation is considered:

$\dot{x}(t)=A x(t)+F f(x(t), t)+A_{d} x(t-\tau)+B[u(t)+d(t)]$,

where $x(t) \in R^{n}, u(t) \in R^{m}$ are the state and the control input, respectively. $A \in R^{n \times n}, B \in R^{n \times m}$, and $A_{d} \in R^{n \times n}$ are the coefficient matrices, satisfying $\operatorname{rank}(B)=m . F$ is the corresponding weighting matrix, $f(x(t), t)$ is nonlinear function which is supposed to satisfy bounded conditions described as Assumption 1. $d(t)$ is a vector of sinusoidal disturbance and $\tau$ is the delay time. Such a model can also represent a wider class of time-delay system compared with papers $[6,9,14]$.

Assumption 1. For any $x_{j}(t) \in R^{n}, j=1,2$ nonlinear functions $f(x, t)$ satisfy

$$
\begin{gathered}
f(0, t)=0, \\
\left\|f\left(x_{1}(t), t\right)-f\left(x_{2}(t), t\right)\right\| \leq\left\|U\left(x_{1}(t)-x_{2}(t)\right)\right\|,
\end{gathered}
$$

where $U$ is the given constant weighting matrix.

Similar to the output regulation theory, DOBC strategy $[9,15]$, each unknown external disturbance $d_{i}(i=$ $1,2, \ldots, m)$ is supposed to be generated by an exogenous system described by

$$
\begin{aligned}
& \dot{w}_{i}=\Gamma_{i} w_{i}, \\
& d_{i}=V_{i} w_{i},
\end{aligned}
$$

where $\left(\Gamma_{i}, V_{i}\right)$ is uniformly observable. To show the main ideology of our paper, suppose $w_{i} \in R^{2}$ and the linear uncertain matrix $\Gamma_{i} \in R^{2 \times 2}$. For sake of simplicity, $\left(\Gamma_{i}, V_{i}\right)$ has observable canonical form, which can be expressed as follows:

$$
\Gamma_{i}=\left[\begin{array}{cc}
0 & 1 \\
-W_{i} & 0
\end{array}\right], \quad V_{i}=\left[\begin{array}{ll}
1 & 0
\end{array}\right], \quad i=1, \ldots, m,
$$

where $W_{i}$ is parameter characteristics related to disturbance frequency; different from the present work, we consider $W_{i}$ to be uncertain constant values, for the sake of simplicity, denote that

$$
W=\left[\begin{array}{c}
W_{1} \\
\vdots \\
W_{n}
\end{array}\right], \quad \Theta=\left[\begin{array}{c}
\Theta_{1} \\
\vdots \\
\Theta_{n}
\end{array}\right], \quad \Xi=\left[\begin{array}{c}
\Xi_{1} \\
\vdots \\
\Xi_{n}
\end{array}\right],
$$

where $\Theta_{i}$ and $\Xi_{i}$ represent precise and unknown part of $W_{i}$, respectively, that is, $W_{i}=\Theta_{i}+\Xi_{i}$. In application, many kinds of disturbances in engineering can be described by this model, for example, the control of aircraft control [9], magnetic bearing control [16], robotic systems [14], and so forth.

In the conventional DOBC strategy $[8,9,11,14,17]$, the $\Gamma_{i}$ in disturbance must be known in advance. This condition is so strict for reason that the disturbances acting on a system are difficult to be modeled precisely in general. Up to now, there is no related method discussing the uncertain disturbances estimation problem subject to time delay. This is the major hurdle that mostly impedes the further research and application in DOBC and other disturbance rejection research.

In this paper, we will derive the relation between the uncertain parameters $\Gamma$ and $d(t)$, according to which the exogenous disturbance may be expressed as nonlinear functions including precise part and uncertain part. The control problem considered will be solved by means of DOBC combined with adaptive control (DOBC + adaptive) such that the proposed controller can achieve arbitrary disturbance attenuation.

\section{Nonlinear Disturbance Observer}

The disturbance parameters are inaccessible in this state timedelay system (1), so it is difficult to construct the disturbance observer with traditional ways directly as in $[9,14]$. In this section, we first design the auxiliary observer for nonlinear vector $\xi$ with time delay. After an appropriate coordinate transformation, the disturbance $d$ may be formulated as a parametric uncertain function. According to (2), $d$ in (1) can be expressed as follows:

$$
\begin{aligned}
& \dot{w}=\Gamma w, \\
& d=V w,
\end{aligned}
$$

where $w \in R^{2 m}, \Gamma \in R^{2 m \times 2 m}$, and

$$
\Gamma=\left[\begin{array}{ccc}
\Gamma_{1} & 0 & 0 \\
0 & \ddots & 0 \\
0 & 0 & \Gamma_{n}
\end{array}\right], \quad V=\left[\begin{array}{ccc}
V_{1} & 0 & 0 \\
0 & \ddots & 0 \\
0 & 0 & V_{n}
\end{array}\right] .
$$


In this section, we suppose that $f(x(t), t)$ is given and Assumption 1 holds. When all states of the system are available, it is unnecessary to estimate the states, then only the estimation of the disturbance need to be concerned. Construct an auxiliary MIMO nonlinear system as follows:

$$
\begin{gathered}
\xi=\nu(t)+\psi, \\
\dot{v}=G(\nu(t)+\psi) \\
-L \check{B}\left(A x+F f(x(t), t)+A_{d} x(t-\tau)+B u(t)\right), \\
\psi=L \check{B} x(t),
\end{gathered}
$$

where

$$
\xi=\left[\begin{array}{c}
\xi_{1} \\
\xi_{2} \\
\vdots \\
\xi_{n}
\end{array}\right], \quad \xi_{i}=\left[\begin{array}{c}
\xi_{i 1} \\
\xi_{i 2}
\end{array}\right]
$$

$G$ and $L$ are given constant matrices in form of

$$
G=\left[\begin{array}{ccc}
G_{1} & 0 & 0 \\
0 & \ddots & 0 \\
0 & 0 & G_{n}
\end{array}\right], \quad L=\left[\begin{array}{ccc}
L_{1} & 0 & 0 \\
0 & \ddots & 0 \\
0 & 0 & L_{n}
\end{array}\right]
$$

where

$$
L_{i}=\left[\begin{array}{l}
0 \\
1
\end{array}\right], \quad G_{i}=\left[\begin{array}{cc}
0 & 1 \\
-g_{i 1} & -g_{i 2}
\end{array}\right],
$$

$G_{i}$ is Hurwitz by selection of $g_{i 1}$ and $g_{i 2}$. Considering $\operatorname{rank}(B)=m$, there exists pseudoinverse $\check{B}$ such that $\check{B} B=I$, so system (8) can be transformed as

$$
\dot{\xi}=G \xi+L d .
$$

Comparing (6) with (12) yields

$$
\left[\begin{array}{c}
\dot{\xi} \\
\dot{w}
\end{array}\right]=\left[\begin{array}{cc}
G & L V \\
0 & \Gamma
\end{array}\right]\left[\begin{array}{c}
\xi \\
w
\end{array}\right] .
$$

Lemma 2. For system (6), if $G_{i}$ and $L_{i}$ have form of (11) and guarantee

$$
\left(g_{i 1}-W_{i}\right)^{2}+g_{i 2}^{2} W_{i} \neq 0
$$

in global region of $W_{i}$, then there exists an invertible constant matrix $\Pi_{i}$ such that

$$
\dot{\xi}_{i}+\Pi_{i} \dot{w}_{i}=G_{i}\left(\xi_{i}+\Pi_{i} w_{i}\right) .
$$

Proof. Considering an invertible matrix

$$
P_{i}^{-1}=\left[\begin{array}{cc}
I^{2 \times 2} & \Pi_{i} \\
0 & I^{2 \times 2}
\end{array}\right],
$$

where

$$
\Pi_{i}=\frac{\left[\begin{array}{cc}
-\left(g_{i 1}-W_{i}\right) & g_{i 2} \\
-W_{i} g_{i 2} & -\left(g_{i 1}-W_{i}\right)
\end{array}\right]}{\left(g_{i 1}-W_{i}\right)^{2}+g_{i 2}^{2} W_{i}},
$$

it is obvious that if (14) is satisfied, then $\Pi_{i}$ is invertible in global region of $W_{i}$; furthermore it can be derived that

$$
\Pi_{i}^{-1}=\left[\begin{array}{cc}
-g_{i 1}+W_{i} & -g_{i 2} \\
W_{i} g_{i 2} & -g_{i 1}+W_{i}
\end{array}\right] .
$$

According to (13), notice that

$$
\left[\begin{array}{c}
\dot{\xi}_{i} \\
\dot{w}_{i}
\end{array}\right]=\left[\begin{array}{cc}
G_{i} & V_{i} L_{i} \\
0 & \Gamma_{i}
\end{array}\right]\left[\begin{array}{c}
\xi_{i} \\
w_{i}
\end{array}\right]
$$

we can define the following coordinate transformation

$$
\left[\begin{array}{c}
\bar{\xi}_{i} \\
\bar{w}_{i}
\end{array}\right]=P_{i}^{-1}\left[\begin{array}{c}
\xi_{i} \\
w_{i}
\end{array}\right]
$$

Combining (16) with (20) yields

$$
\left[\begin{array}{c}
\dot{\bar{\xi}}_{i} \\
\dot{\bar{w}}_{i}
\end{array}\right]=P_{i}^{-1}\left[\begin{array}{cc}
G_{i} & V_{i} L_{i} \\
0 & \Gamma_{i}
\end{array}\right] P_{i}\left[\begin{array}{c}
\bar{\xi}_{i} \\
\bar{w}_{i}
\end{array}\right] .
$$

After calculation, it can be verified that

$$
P_{i}^{-1}\left[\begin{array}{cc}
G_{i} & L_{i} V_{i} \\
0 & \Gamma_{i}
\end{array}\right] P_{i}=\left[\begin{array}{cc}
G_{i} & 0 \\
0 & \Gamma_{i}
\end{array}\right]
$$

Thus (15) can be got directly following (21) and (22).

Based on Lemma 2, we can give another form of $d_{i}$ as

$$
d_{i}(t)=V_{i} w_{i}(t)=-\bar{V}_{i}(t) \xi_{i}(t)+\bar{V}_{i}(t) \bar{\xi}_{i}(t),
$$

where

$$
\begin{gathered}
w_{i}(t)=-\Pi_{i}^{-1} \xi_{i}(t)+\Pi_{i}^{-1} \bar{\xi}_{i}(t), \quad \bar{V}_{i}=V_{i} \Pi_{i}^{-1}, \\
\bar{\xi}_{i}=\xi_{i}+\Pi_{i} w_{i}
\end{gathered}
$$

and satisfy

$$
\dot{\bar{\xi}}_{i}=G_{i} \bar{\xi}_{i}
$$

Similarly, $d$ can be rewritten as

$$
d=\bar{V} \xi+\bar{V} \bar{\xi}
$$

where

$$
\bar{V}=\left[\begin{array}{ccc}
V_{1} \Pi_{1}^{-1} & 0 & 0 \\
0 & \ddots & 0 \\
0 & 0 & V_{m} \Pi_{m}^{-1}
\end{array}\right], \quad \bar{\xi}=\left[\begin{array}{c}
\bar{\xi}_{1} \\
\vdots \\
\bar{\xi}_{m}
\end{array}\right],
$$

according to (10) and (25), $\bar{\xi}$ satisfies

$$
\dot{\bar{\xi}}=G \bar{\xi} .
$$

From Lemma 2, we have given another form of $d$ through auxiliary observer $\xi$, and can construct observer of $d$ as

$$
\widehat{d}=\bar{V} \xi
$$


thus the proposed method will exhibit classic DOBC property.

Notice that $\bar{V}$ in observer $\widehat{d}$ cannot be implemented directly as the $W_{i}$ is uncertain. To show it clearly, we divide $\widehat{d}$ into two parts. One part is a precise value that can be predicted and the other is uncertain constant parameter multiplied by a known nonlinear term. For the sake of simplicity, denote

$$
\begin{aligned}
\bar{V} \xi= & {\left[\begin{array}{ccc}
V_{1} & 0 & 0 \\
0 & \ddots & 0 \\
0 & 0 & V_{n}
\end{array}\right]\left[\begin{array}{c}
-\Pi_{1}^{-1} \xi_{1} \\
-\Pi_{2}^{-1} \xi_{2} \\
\vdots \\
-\Pi_{n}^{-1} \xi_{n}
\end{array}\right] } \\
= & {\left[\begin{array}{c}
\left(-g_{11}+\Theta_{11}\right) \xi_{11}+\left(\Theta_{12}-g_{12}\right) \xi_{12} \\
\vdots \\
\left(-g_{m 1}+\Theta_{m 1}\right) \xi_{m 1}+\left(\Theta_{m 2}-g_{m 2}\right) \xi_{n 2}
\end{array}\right] } \\
& +\left[\begin{array}{ccc}
\xi_{11} & \ddots \\
& & \xi_{m 1}
\end{array}\right]\left[\begin{array}{c}
\Xi_{1} \\
\vdots \\
\Xi_{n}
\end{array}\right] .
\end{aligned}
$$

A notable property of (29) is that uncertain sinusoidal $d$ can be expressed in form of parametric uncertainty. So, we need not estimate the upper bounds of $d$ as in [18-21].

\section{DOBC with Stability Analysis}

After substituting (26) into system (1), we have

$$
\begin{aligned}
\dot{x}(t)= & A x(t)+F f(x(t), t)+A_{d} x(t-\tau) \\
& +B[u(t)+\bar{V} \xi+\bar{V} \bar{\xi}] .
\end{aligned}
$$

For the plants with known nonlinearity, the DOBC+ adaptive strategy can be designed by using the separation principle as follows:

$$
u=K x+u_{o}+u_{a}
$$

where $K$ is the conventional control gain for stabilization, $u_{o}$ and $u_{a}$ are used to reject and attenuate the disturbances known and uncertain parts, respectively, and according to (30) we select

$$
u_{o}=-\left[\begin{array}{c}
\left(-g_{11}+\Theta_{11}\right) \xi_{11}+\left(\Theta_{12}-g_{12}\right) \xi_{12} \\
\vdots \\
\left(-g_{m 1}+\Theta_{m 1}\right) \xi_{m 1}+\left(\Theta_{m 2}-g_{m 2}\right) \xi_{m 2}
\end{array}\right] .
$$

Similar to [22], adaptive controller $u_{a}$ is used to compensate unknown part of disturbance $d$ which satisfies

$$
u_{a}=-\bar{\Theta} \widehat{\Xi}
$$

where

$$
\bar{\Theta}=\left[\begin{array}{lll}
\xi_{11} & & \\
& \ddots & \\
& & \xi_{n 1}
\end{array}\right]
$$

and $\widehat{\Xi}$ is estimation of $\Xi$. At last the dynamic system (31) may be rewritten as follows:

$$
\begin{aligned}
\dot{x}(t)= & A x(t)+F f(x(t), t)+A_{d} x(t-d) \\
& +B[K x+\bar{\Theta}(\Xi-\widehat{\Xi})+\bar{V} \bar{\xi}] .
\end{aligned}
$$

At this stage, our objective is to find $K$ such that the closed-loop system (31) with $u=K x$ is asymptotically stable. For the sake of simplifying descriptions, we denote $\operatorname{sym}(M):=M+M^{T}$ and

$$
N_{1}=P_{1}(A+B K)+(A+B K)^{T} P_{1}
$$

Theorem 3. For given $\lambda>0$, if (14) can be guaranteed and there exist $Q_{1}>0, P_{1}>0, E=S^{-1}>0$ and $R_{1}$ satisfying

$$
\Omega=\left[\begin{array}{ccccc}
\operatorname{Sym}\left(\mathrm{AQ}_{1}+\mathrm{BR}_{1}\right) & A_{d} E & F_{1} & \mathrm{Q}_{1} & U Q_{1} \\
* & -E & 0 & 0 & 0 \\
* & * & -\frac{1}{\lambda_{1}^{2}} I & 0 & 0 \\
* & * & * & -E & 0 \\
* & * & * & * & *-\frac{1}{\lambda_{1}^{2}} I
\end{array}\right]<0,
$$

then under DOBC law (32) and adaptive dynamic

$$
\dot{\widehat{\Xi}}=\gamma_{1} \bar{\Theta}^{T} B^{T} P x, \quad\left(\gamma_{1}>0\right)
$$

the closed-loop system (36) with gain $K=R_{1} Q_{1}^{-1}$ is asymptotically stable.

Proof. Denote $V(x(t), \bar{\xi}(t), \widetilde{\Xi}(t), t)=V_{1}(x(t), t)+V_{2}(\bar{\xi}(t), t)+$ $V_{3}(\widetilde{\Xi}(t), t)$, where

$$
\begin{gathered}
V_{1}(x(t), t)=x(t)^{T} P x(t) \\
+\frac{1}{\lambda^{2}} \int_{0}^{t}\left(\|U x(\sigma)\|^{2}-\|f(x(\sigma), \sigma)\|^{2}\right) d \sigma \\
\quad+\int_{t-\tau}^{t} x^{T}(\sigma) S x(\sigma) d \sigma \\
V_{2}(\bar{\xi}(t), t)=\gamma_{2} \bar{\xi}(t)^{T} P_{2} \bar{\xi}(t), \quad\left(\gamma_{2}>0\right) \\
V_{3}(\Xi(t), t)=\gamma_{1}^{-1} \widetilde{\Xi}^{T} \widetilde{\Xi} .
\end{gathered}
$$


Along with the trajectories of (36) and (39), firstly it can be verified that

$$
\begin{aligned}
\dot{V}_{1}(x(t), t)+\dot{V}_{3}(\widetilde{\Xi}, t) \\
=\dot{x}(t)^{T} P x(t)+x(t)^{T} P \dot{x}(t) \\
+\frac{1}{\lambda^{2}}\left(\|U x(t)\|^{2}-\|f(x(t), t)\|^{2}\right) \\
+x^{T}(t) S x(t)-x^{T}(t-\tau) S x(t-\tau)+2 x^{T}(t) P B \bar{V} \bar{\xi} \\
\leq \\
\left.+A x(t)+F f(x(t), t)+A_{d} x(t-\tau)\right)^{T} P x(t) \\
+x^{T}(t) P\left(A x(t)+F f(x(t), t)+A_{d} x(t-\tau)\right) \\
+\frac{1}{\lambda^{2}} x^{T}(t) U^{T} U x(t)-\frac{1}{\lambda^{2}} f^{T}(x(t), t) f(x(t), t) \\
+x^{T}(t) S x(t)-x^{T}(t-\tau) S x(t-\tau)+2 x^{T} P B \bar{V} \bar{\xi} \\
=\left[\begin{array}{c}
x(t) \\
x(t-\tau) \\
f(x(t), t)
\end{array}\right]^{T}\left[\begin{array}{c}
x(t) \\
x(t-\tau) \\
\Omega_{1}(x(t), t)
\end{array}\right]+2 x^{T} P B \bar{V} \bar{\xi},
\end{aligned}
$$

where

$$
\Omega_{1}=\left[\begin{array}{ccc}
N_{1}+S+\frac{1}{\lambda_{1}^{2}} U_{1}^{T} U_{1} & P A_{d} & P F_{1} \\
* & -S & 0 \\
* & * & -\frac{1}{\lambda_{1}^{2}} I
\end{array}\right] .
$$

Premultiplied and postmultiplied simultaneously by diag $\{Q, I, I\}, \Omega_{1}<0$ is equivalent to $\Omega_{2}<0$, where

$$
\Omega_{2}=\left[\begin{array}{ccc}
\operatorname{Sym}\left(A Q_{1}+B R_{1}\right)+Q_{1} S Q_{1}+\frac{1}{\lambda_{1}^{2}} Q_{1} U_{1}^{T} U_{1} Q_{1} & A_{d} & F_{1} \\
* & -S & 0 \\
* & * & -\frac{1}{\lambda_{1}^{2}} I
\end{array}\right]
$$

Based on Schur complement, it can be seen that $\Omega_{2}<0$ is equivalent to $\Omega_{3}<0$ and

$$
\Omega_{3}=\left[\begin{array}{ccccc}
\operatorname{Sym}\left(A Q_{1}+B R_{1}\right) & A_{d} E & F_{1} & Q_{1} & U_{1} Q_{1} \\
* & -S & 0 & 0 & 0 \\
* & * & -\frac{1}{\lambda_{1}^{2}} I & 0 & 0 \\
* & * & * & -S^{-1} & 0 \\
* & * & * & * & *-\frac{1}{\lambda_{1}^{2}} I
\end{array}\right] .
$$

Premultiplied and postmultiplied simultaneously by diag $\{I, E, I, I, I\}, \Omega_{3}<0$ is equivalent to $\Omega<0$. That is to say that if (38) upholds, there exists constant $\alpha_{1}>0$ such that

$$
\dot{V}_{1}(x(t), t)+\dot{V}_{3}(\widetilde{\Xi}, t)<-\alpha_{1}\left\|\left[\begin{array}{c}
x(t) \\
x(t-\tau) \\
f(x(t), t)
\end{array}\right]\right\|^{2}+2 x^{T} P B \bar{V} \bar{\xi} .
$$

Together with the definition of $G$ in (10), we can find that $\alpha_{2}>$ 0 satisfies

$$
G^{T} P_{2}+P_{2} G+\alpha_{2}<0
$$

Furthermore, there exists $\gamma_{3}>0$ depending on $P_{1}$ such that for any $x$ and $\bar{\xi}$

$$
2 x^{T} P_{1} B \bar{V} \bar{\xi} \leq \gamma_{3}\|x\|\|\bar{\xi}\|
$$

After substituting (45), (46), and (49), derivative along Lyapunov function candidate is given by

$$
\begin{aligned}
\dot{V}(x, \bar{\xi}, \widetilde{\Xi}) & \leq-\alpha_{1}\left\|\left[\begin{array}{c}
x(t) \\
x(t-\tau) \\
f(x(t), t)
\end{array}\right]\right\|^{2}+\gamma_{3}\|x\|\|\bar{\xi}\|-\alpha_{2} \gamma_{2}\|\bar{\xi}\|^{2} \\
& \leq-\alpha_{1}\|[x(t)]\|^{2}+\gamma_{3}\|x\|\|\bar{\xi}\|-\alpha_{2} \gamma_{2}\|\bar{\xi}\|^{2} .
\end{aligned}
$$

The right part of the above inequality can be regarded as a polynomial with respect to two variables $\|x\|$ and $\|\bar{\xi}\|$. Thus for all $\|x\|$ and $\|\bar{\xi}\|, \dot{V}(x, \bar{\xi}, \widetilde{\Xi}) \leq 0$ holds if there exists a group of parameters $\gamma_{i}(i=2,3)$ satisfying

$$
2 \sqrt{\alpha_{1} \alpha_{2} \gamma_{2}} \geq \gamma_{3}
$$

The disturbance-observer-based control design procedure can be summarized as follows.

Step 1. Select weighting matrices $G$ and $L$ with form of (10) and (11), apply $G$ and $L$ into (8) to calculate auxiliary vector.

Step 2. According to (26), give another form of disturbance represented by auxiliary vector.

Step 3. Design time-delay feedback controller $K X$ based on Theorem 3, apply controller into (32), then DOBC + adaptive control can be realized.

\section{Simulation}

To show the efficiency of the proposed scheme, let us consider the continuous time-delay models under the proposed $\mathrm{DOBC}+$ adaptive scheme. The longitudinal dynamics of A4D aircraft at a flight condition of $15000 \mathrm{ft}$ altitude and 0.9 Mach can be given by (1). The meaning and significance of the parameters are the same as in $[6,9]$, where $x_{1}(t)$ is the forward velocity $(\mathrm{ft} / \mathrm{s}), x_{2}(t)$ is the angle of attack $(\mathrm{rad}), x_{3}(t)$ is the pitching velocity $(\mathrm{rad} / s), x_{4}(t)$ is the pitching angle ( $\left.\mathrm{rad}\right)$, and 
$u(t)$ is elevator deflection (deg) and coefficient matrices:

$$
\begin{aligned}
& A=\left[\begin{array}{cccc}
-0.0605 & 32.37 & 0 & 32.2 \\
-0.00014 & -1.475 & 1 & 0 \\
-0.0111 & -34.72 & -2.793 & 0 \\
0 & 0 & 1 & 0
\end{array}\right], \\
& B=\left[\begin{array}{c}
0 \\
-1.1064 \\
-33.8 \\
0
\end{array}\right] \\
& A_{d}=\left[\begin{array}{cccc}
0.09 & 0.03 & 0.03 & 1.03 \\
0.002 & 0.04 & 0.07 & -0.0034 \\
-0.004 & -0.04 & 0.03 & -0.001 \\
-0.0004 & -0.014 & 0.008 & 0.03
\end{array}\right] \text {, } \\
& F=\left[\begin{array}{c}
0 \\
0 \\
50 \\
0
\end{array}\right]
\end{aligned}
$$

Similar to [9], it is supposed that nonlinearity and/or uncertainty $f(x(t), t)=\sin (2 \pi 5 t) x_{2}(t)$, state delay time $\tau=2$, and set $U=\operatorname{diag}\{0100\}$ guarantee $\|f(x(t), t)\| \leq\|U f(x(t), t)\|$. Paper $[9,14]$ pointed out that if the frequency is perturbed, the pure DOBC approach will be unavailable because the disturbances cannot be rejected accurately. In order to investigate further, it has been considered that uncertainties exist in such an exogenous model for the disturbance in (3). That is,

$$
d=25[\sin ((1+\Xi) t)],
$$

where frequency perturbations $\Xi_{11}=\Xi_{21}=4$. Set

$$
G=\left[\begin{array}{cc}
0 & 1 \\
-4 & -4
\end{array}\right], \quad \gamma_{1}=10000
$$

It is noted that the selection of $\lambda$ is tradeoff and we select $\lambda=$ 0.8 , and the initial value of the disturbance is 0 . When the full states can be measured, applying the approach in Theorem 3, when DOBC law is applied in system (31), the corresponding parameter in (32) can be gotten that

$$
K=\left[\begin{array}{llll}
9.9316 & 530.5033 & 1.7402 & 552.7417
\end{array}\right] .
$$

Figure 1 plots the estimation error of the uncertain disturbances with traditional DO $[9,11,14]$. The results show that if system suffers uncertain disturbance, it may bring large disturbance estimation error, and the control performance is deteriorated (Figure 2). Figures 3 and 4 demonstrate the system performance using the proposed DOBC + adaptive schemes, obviously the system output converges to zero with sufficiently small steady error. The results show that although there exists uncertainty in the disturbance parameters, the disturbance rejection performance is improved and enhanced system responses can be achieved.

\section{Conclusion}

The DOBC strategy is extended to the uncertain disturbance rejection problem by combining with adaptive control. We

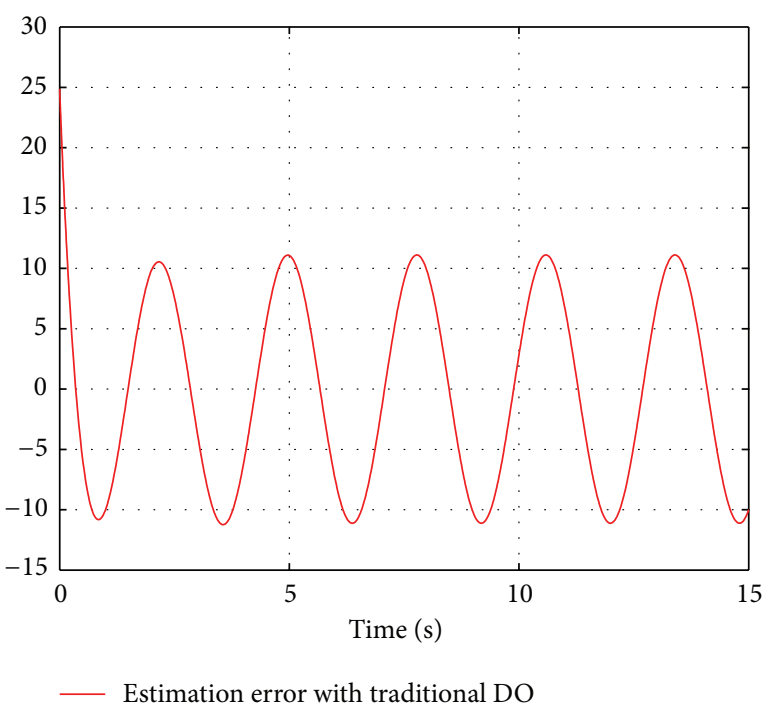

FIGURE 1: Estimation error using traditional DO.

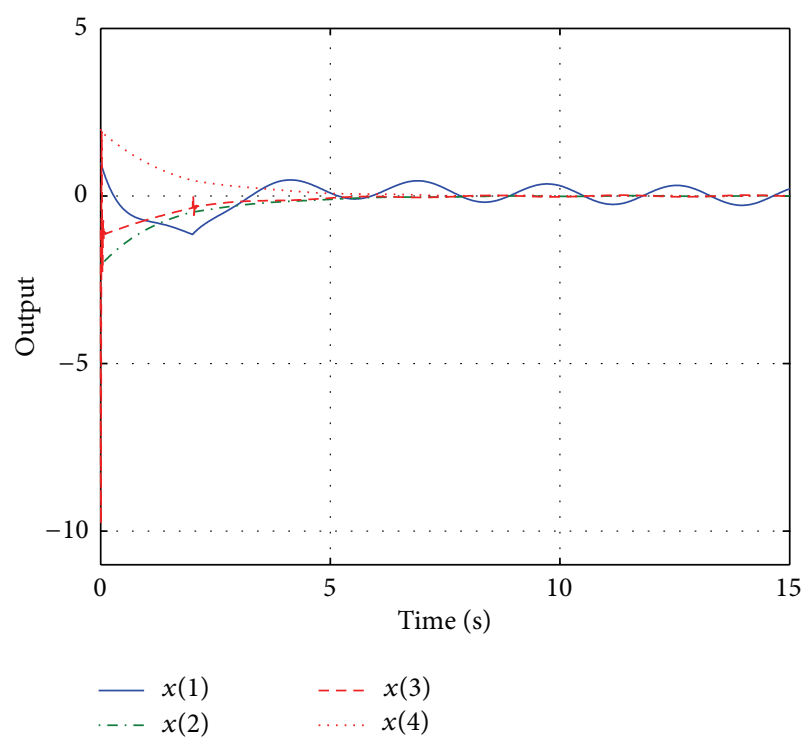

Figure 2: System output using traditional DOBC.

first construct the auxiliary observer for $d(t)$ with uncertain parameters, and then the exogenous disturbance may be divided into two parts. One part is a precise term which can be compensated by a feed-forward controller, and the other can be expressed as uncertain constant parameter multiplied by a known nonlinear term; an adaptive controller is adopted to compensate the effect of the second part. Simulations on an aircraft model demonstrate the advantages of the proposed scheme. However, if there exist multiple disturbances and unmodeled dynamics in the system as in $[10,11]$, the situation turns to be more complicated, and further research is required in the future. 


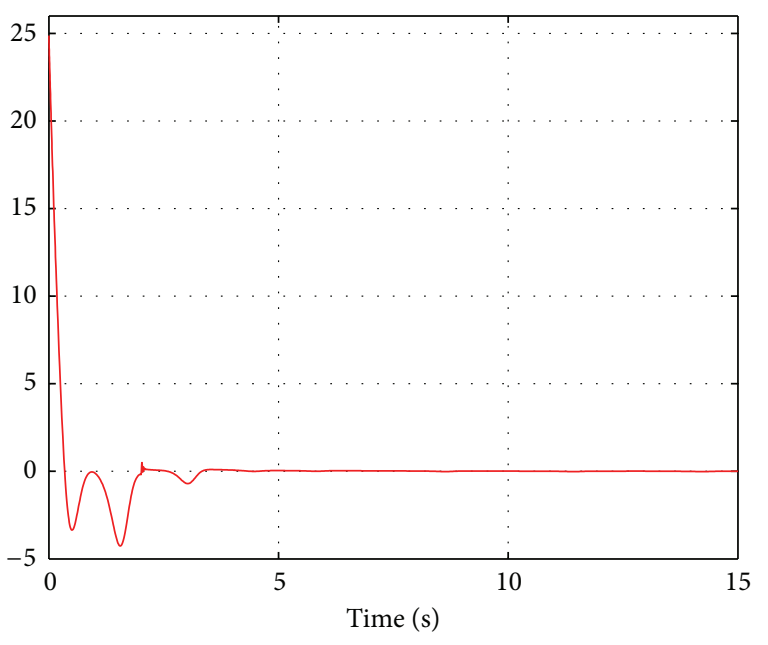

_ Estimation error with our DO

FIGURE 3: Estimation error using DO proposed in this paper.

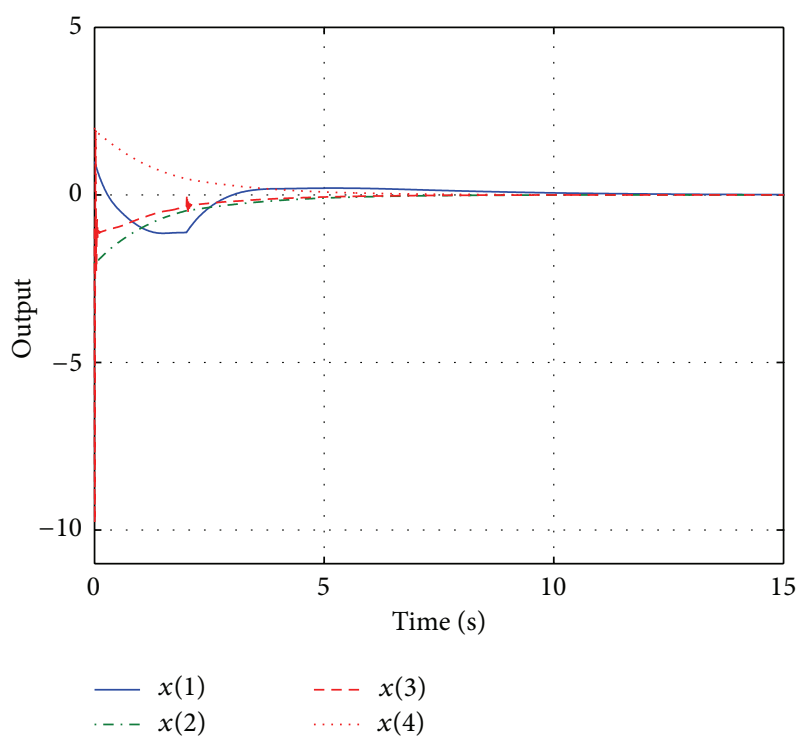

FIGURE 4: System output using DOBC + adaptive.

\section{Acknowledgments}

This work was supported by the National Natural Science Foundation of China under Grant no. 61203049 and Scholar Doctor Priming Foundation (20122048).

\section{References}

[1] H. Gao and T. Chen, "New results on stability of discrete-time systems with time-varying state delay," IEEE Transactions on Automatic Control, vol. 52, no. 2, pp. 328-334, 2007.

[2] M. S. Mahmoud, Y. Shi, and H. N. Nounou, "Resilient observerbased control of uncertain time-delay systems," International Journal of Innovative Computing, Information and Control, vol. 3, no. 2, pp. 407-418, 2007.
[3] X. M. Sun, J. Zhao, and W. Wang, "Two design schemes for robust adaptive control of a class of linear uncertain neutral delay systems," International Journal of Innovative Computing, Information and Control, vol. 3, no. 2, pp. 385-396, 2007.

[4] H. Du, J. Lam, and K. Y. Sze, " $H_{\infty}$ disturbance attenuation for uncertain mechanical systems with input delay," Transactions of the Institute of Measurement and Control, vol. 27, no. 1, pp. 37-52, 2005.

[5] Y. S. Lee, Y. S. Moon, W. H. Kwon, and P. G. Park, "Delaydependent robust $H_{\infty}$ control for uncertain systems with a state-delay," Automatica, vol. 40, no. 1, pp. 65-72, 2004.

[6] X. Wei, H. Zhang, and L. Guo, "Saturating composite disturbance-observer-based control and $H_{\infty}$ control for discrete time-delay systems with nonlinearity," International Journal of Control, Automation and Systems, vol. 7, no. 5, pp. 691-701, 2009.

[7] L. Guo, C. Feng, and W. Chen, "A survey of disturbanceobserver-based control for dynamic nonlinear system," Dynamics of Continuous Discrete and Impulsive Systems. Series B, vol. 13E, pp. 79-84, 2006.

[8] W. H. Chen, D. J. Ballance, P. J. Gawthrop, and J. O'Reilly, "A nonlinear disturbance observer for robotic manipulators," IEEE Transactions on Industrial Electronics, vol. 47, no. 4, pp. 932-938, 2000.

[9] L. Guo and W. H. Chen, "Disturbance attenuation and rejection for systems with nonlinearity via DOBC approach," International Journal of Robust and Nonlinear Control, vol. 15, no. 3, pp. 109-125, 2005.

[10] L. Guo and X. Y. Wen, "Hierarchical anti-distance adaptive control for non-linear systems with composite disturbances and applications to missile systems," Transactions of the Institute of Measurement and Control, vol. 33, no. 8, pp. 942-956, 2011.

[11] X. Wei and L. Guo, "Composite disturbance-observer-based control and $H_{\infty}$ control for complex continuous models," International Journal of Robust and Nonlinear Control, vol. 20, no. 1, pp. 106-118, 2010.

[12] H. Wu, "Adaptive robust state observers for a class of uncertain nonlinear dynamical systems with delayed state perturbations," IEEE Transactions on Automatic Control, vol. 54, no. 6, pp. 14071412, 2009.

[13] J. Yang, S. Li, X. Chen, and Q. Li, "Disturbance rejection of ball mill grinding circuits using DOB and MPC," Powder Technology, vol. 198, no. 2, pp. 219-228, 2010.

[14] W. H. Chen, "Disturbance observer based control for nonlinear systems," IEEE/ASME Transactions on Mechatronics, vol. 9, no. 4, pp. 706-710, 2004

[15] A. Isidori and C. I. Byrnes, "Output regulation of nonlinear systems," IEEE Transactions on Automatic Control, vol. 35, no. 2, pp. 131-140, 1990.

[16] T. Mizuno and T. Higuchi, "Design of magnetic bearing controllers based on disturbance estimation," in Proceedings of the the 2nd International Symposium on Magnetic Bearings, pp. 281288, NISSEI EBLO, Tokyo, Japan, 1990.

[17] J. Yang, W. H. Chen, and S. H. Li, "Nonlinear disturbance observer based robust control for systems with mismatched disturbances/uncertainties," IET Control Theory and Application, vol. 5, no. 18, pp. 2053-2062, 2011.

[18] J. Back and H. Shim, "Adding robustness to nominal outputfeedback controllers for uncertain nonlinear systems: a nonlinear version of disturbance observer," Automatica, vol. 44, no. 10, pp. 2528-2537, 2008. 
[19] X. Chen, C. Y. Su, and T. Fukuda, "A nonlinear disturbance observer for multivariable systems and its application to magnetic bearing systems," IEEE Transactions on Control Systems Technology, vol. 12, no. 4, pp. 569-577, 2004.

[20] J. H. She, Y. Ohyama, and M. Nakano, "A new approach to the estimation and rejection of disturbances in servo systems," IEEE Transactions on Control Systems Technology, vol. 13, no. 3, pp. 378-385, 2005.

[21] Z. Y. Yang, H. Tsubakihara, S. Kanae, K. Wada, and C. Y. Su, "A novel robust nonlinear motion controller with disturbance observer," IEEE Transactions on Control Systems Technology, vol. 16, no. 1, pp. 137-147, 2008.

[22] V. O. Nikiforov, "Nonlinear servocompensation of unknown external disturbances," Automatica, vol. 37, no. 10, pp. 1647-1653, 2001. 


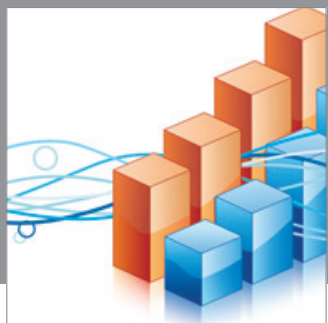

Advances in

Operations Research

mansans

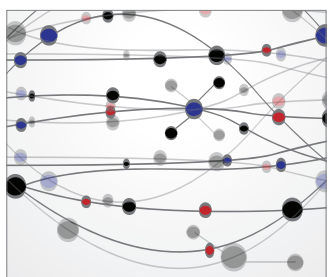

The Scientific World Journal
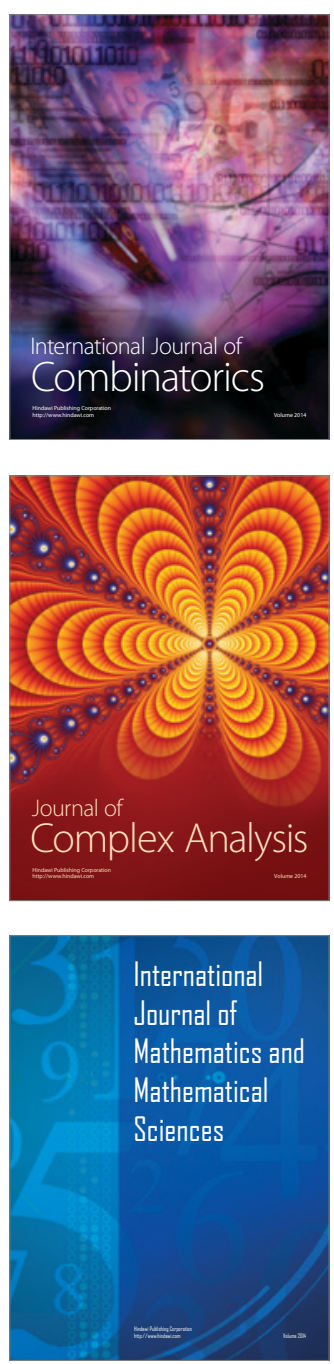
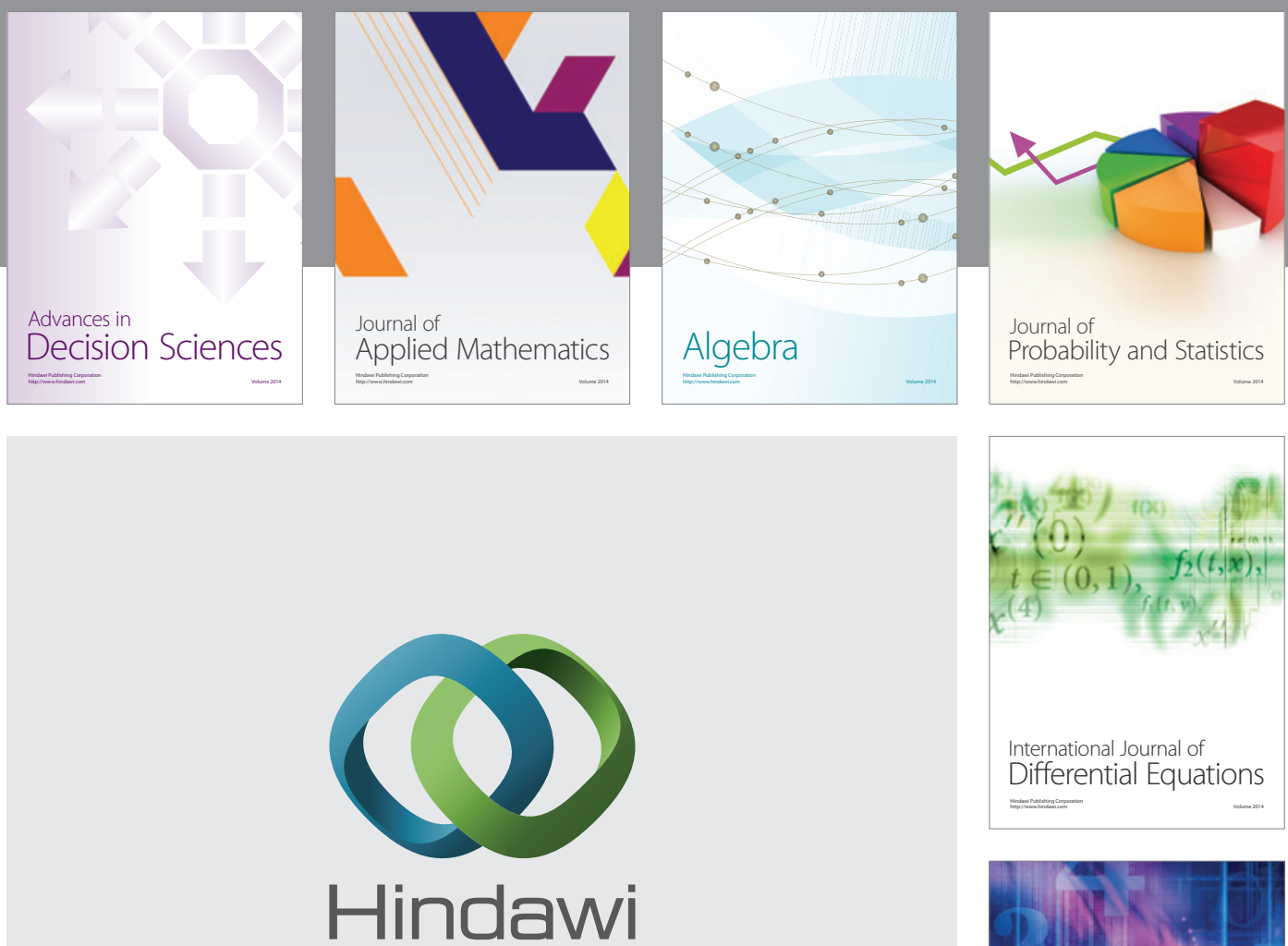

Submit your manuscripts at http://www.hindawi.com
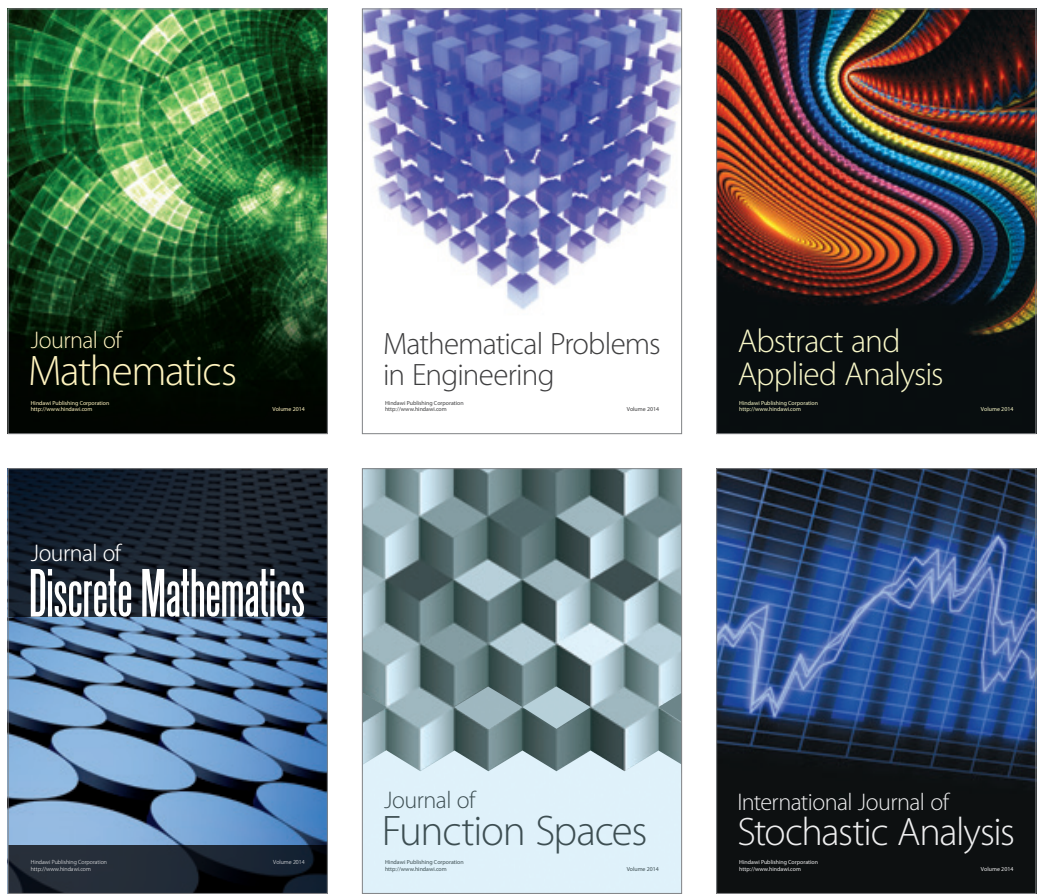

Journal of

Function Spaces

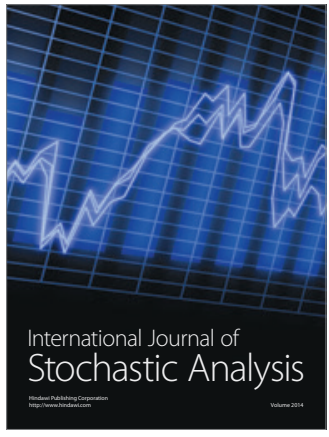

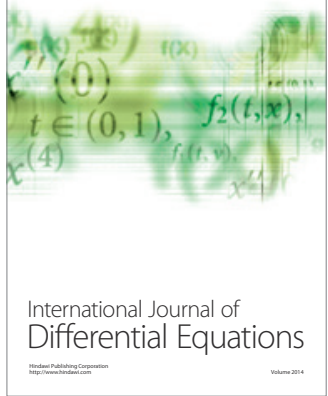
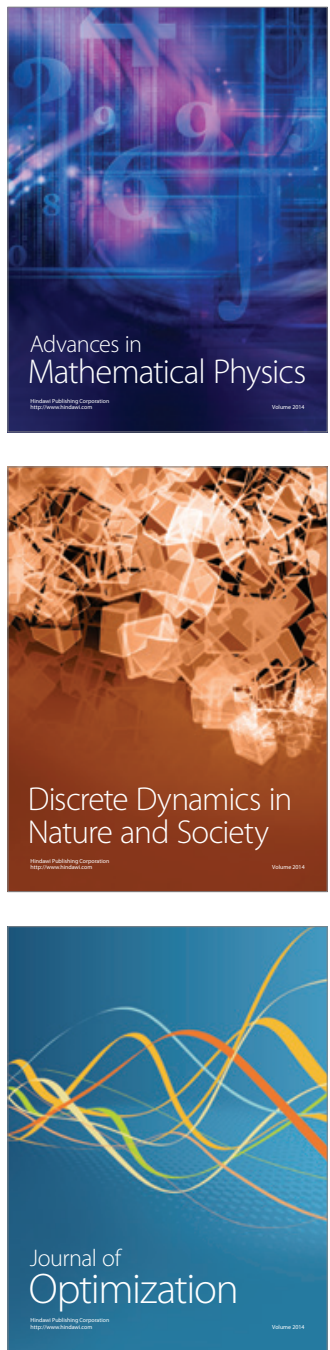